\title{
Experimental realisation of predefined diesel combustion processes using advanced closed-loop combustion control and injection rate shaping
}

International J of Engine Research

\author{
Jan Hinkelbein', Florian Kremer², Matthias Lamping', Thomas Körfer', \\ Joschka Schaub ${ }^{2}$ and Stefan Pischinger ${ }^{2}$
}

\begin{abstract}
In this paper, a combustion control algorithm is presented that, in combination with rate shaping, allows closed-loop control of a cylinder pressure trace. Given this system, it is possible to control the behaviour of the entire combustion process. The paper starts with an explanation of the control algorithm that was developed based on iterative learning control. Consequently, the so-called $\alpha$-process, which comprises a constant pressure rise, is presented as an example of the additional degrees of freedom gained. Based on the exact analysis of experimental results and combustion simulations, the effects of a peak pressure limitation on the emission behaviour of a single-cylinder engine powered by an $\alpha$-process are analysed in detail. The capability of the developed control system to isolate certain effects of ideal combustion processes gives a wide range of possible further investigations. However, for practical applications, the use of injection rate shaping is coupled with high hardware costs. Therefore, an additional concept study regarding the possible realisation of the developed control system with a conventional common-rail injector is presented. In this study, it is shown that injectors without rate-shaping capabilities are able to solve the feedback control problem through multiple injection strategies.
\end{abstract}

\section{Keywords \\ Closed-loop combustion control, injection rate shaping, iterative learning control, combustion process, $\alpha$-process, multiple injection strategies}

Date received: 24 June 2010; accepted: 26 January 2012

\section{Introduction}

In order to analyse and improve the combustion process in a direct-injection diesel engine, different ideal processes, such as the Seiliger process, are well known and have been established in fundamental engine research. While combustion under real operating conditions differs from this theoretical case, it is still desirable to follow these pressure traces as accurately as possible in order to identify and understand the significant characteristics of this specific process. Therefore, a precise and adequate control system is inevitable.

In engine technology, increased introduction of injection systems with rate-shaping capability has been observed. ${ }^{1}$ These injection systems manipulate the injection rates using needle lifts or variations of the injection pressure, which allow direct modulation of the combustion. Improvements of exhaust emissions using rate shaping have already been observed..$^{2-4}$
The present paper outlines the benefits of a combination of injection rate shaping and closed-loop combustion control. Thus, a flexible injection rate-shaping tool (FIRST) developed by FEV GmbH is used (see the later section on injector and system setup). Additionally, a control algorithm that uses iterative learning control (ILC) to perform experimental system inversion is applied. This combination raises the possibility of presetting the whole combustion process by defining the desired process output without knowing the required injection rate beforehand. As an example, the so-called

'FEV GmbH, Aachen, Germany

${ }^{2}$ Institute for Combustion Engines, RWTH Aachen University, Germany

\section{Corresponding author:}

Stefan Pischinger, Institute for Combustion Engines (VKA), RWTH

Aachen University, Schinkelstraße 8, D-52062 Aachen, Germany.

Email: office@vka.rwth-aachen.de 
$\alpha$-process, which describes a constant pressure rise during the combustion, is introduced. The difference between a pure $\alpha$-process and an $\alpha$-process with peak pressure limitation (PPL) is analysed using experimental results of a single-cylinder engine.

Furthermore, a parameter study is presented that analyses the potential of multiple injection strategies to perform rate shaping and obtain predefined pressure traces without an injector with rate-shaping capabilities. The required injector dynamics are discussed at the end of this paper.

\section{ILC}

ILC algorithms were developed in 1984 to improve the operation of robots. ${ }^{5}$ Later on, ILC was used to control a wide variety of plants, such as vibration test benches for fatigue tests, ${ }^{6}$ and cyclic production processes, such as extruders. ${ }^{7}$ However, the application of cylinder pressure control to diesel engines has not yet been observed.

From a mathematical point of view, the ILC illustrates a stepwise experimental process inversion. The actuating variable of the process will be modified from control cycle to control cycle until the desired output value is reached. During one control cycle, an openloop control of the process takes place. The actuating variable for the whole control cycle is calculated offline based on the results of the previous control cycles, and is then used for the actuation of the system. By using the feedback from previous control cycles, a closedloop control across control cycles is generated. ${ }^{6}$ ILC is obtained with an open-loop control in combination with a memory element. The analysis of the memory as a whole takes place between the control cycles (i.e. a pause must be available between each control cycle).

In Figure 1, the structure of the ILC is shown. The central memory $\mathrm{z}^{-1}$ separates the actuating variables of two consecutive control cycles $\underline{u}_{k}$ and $\underline{u}_{k+1}$ from each other before they are applied to the plant $\underline{\mathrm{G}}$. The feedback $y_{k}$ for the generation of the control cycle to control cycle closed-loop control is also shown. The actuation signal for the next control cycle

$$
\underline{u}_{k+1}(i)=\underline{\mathrm{S}} \underline{u}_{k}(i)+\underline{\Gamma} \underline{e}_{k}(i)
$$

is generated from the actuating signal vector of the previous control cycle, which is weighed with the actuating

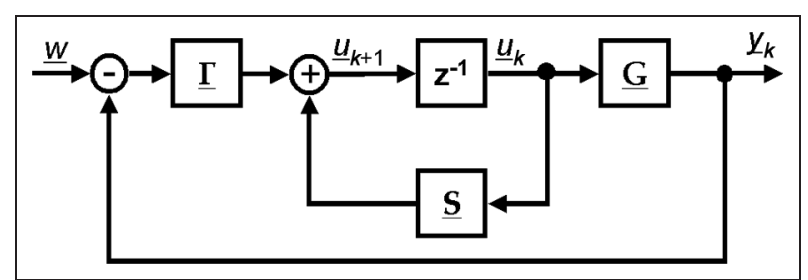

Figure I. Time-domain ILC control structure. variable filter $\underline{S}$, and the control error, which is weighed with the learning operator $\underline{\Gamma}$. The control error

$$
\underline{e}_{k}(i)=\underline{w}-\underline{y}_{k}
$$

is calculated from the setpoint vector $\underline{\mathrm{w}}$ and the feedback of the current control cycle. Equation (1) forms the core of the ILC and is therefore named the learning rule, as the learning procedure is processed here. The learning operator is taken as constant for all control cycles.

Neither the actuating variable filter nor the learning operator includes any system information. ILC can therefore be designed and operated with minimal system knowledge. Furthermore, one of the advantages of ILC is that it can adjust to a time-variant system.

Equation (1) shows that complete learning is only possible when all information from the previous control cycle is taken over to the current control cycle. The actuating variable filter has to be constructed as an identity matrix

$$
\underline{\mathrm{S}}=\underline{\mathrm{I}}
$$

in order to reach full noise suppression and stationary accuracy. A more detailed description of the control algorithm and the specific governor design can be found in Hinkelbein et al. ${ }^{8}$

The control algorithm obtained uses multiple engine cycles (e.g. 20) to generate one control cycle; therefore, the settling time of the ILC algorithm is of the order of several hundred engine cycles, or up to $60 \mathrm{~s}$ for an engine speed of $2280 \mathrm{r} / \mathrm{min}$. This is due to the iterative nature of the algorithm, and shows that it is not feasible for transient engine operation without an additional pre-control methodology. In this paper, the control system is therefore used as a calibration tool for stationary engine operation. The actuating variable filter $\underline{S}$ is designed as the identity matrix. The design of the learning operator $\underline{\Gamma}$ only influences the settling time of the controller, not the stationary results. Therefore, its design will not be shown in detail in this paper. The control error of the control algorithm is always the difference of the cylinder pressure setpoint and the actual measured cylinder pressure of the engine.

\section{Injector and system setup}

The system setup and signal flow is shown in Figure 2. In the single-cylinder engine (see Table 1 for specifications), the cylinder pressure is measured using a piezoelectric pressure transducer. The calculation of the control algorithm is executed in a rapid control prototyping (RCP) system by using the measured crank angle (CA) signal and the amplified pressure transducer signal. The resulting injector voltage (actuating variable of the control algorithm) is amplified by a power stage and then fed to the injector, which injects the fuel into the engine.

The FIRST injector was developed to be able to investigate the full potential of injection rate shaping 


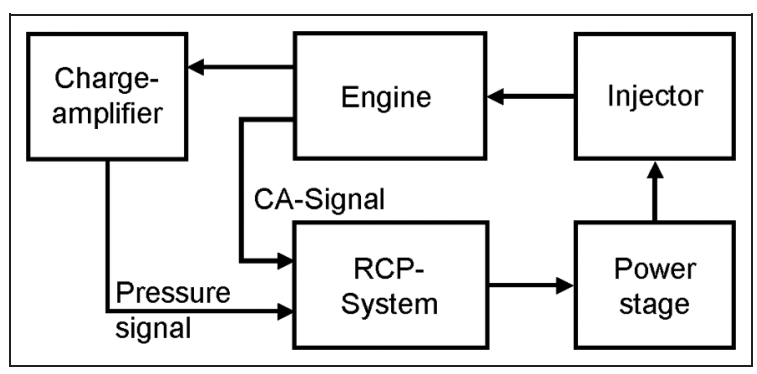

Figure 2. Signal flow and components of the control system (CA: crank angle; RCP: rapid control prototyping).

Table I. Specifications of engine and nozzle.

\begin{tabular}{lc}
\hline Engine & \\
\hline Number of cylinders & 1 \\
Number of valves & 4 \\
Bore $(\mathrm{mm})$ & 81 \\
Stroke $(\mathrm{mm})$ & 88 \\
Displacement $\left(\mathrm{cm}^{3}\right)$ & 453 \\
Compression ratio & 15.8 \\
Swirl (cu/ca @ maximum valve lift) & 1.7 \\
\hline Nozzle & \\
\hline Cone angle $\left({ }^{\circ}\right)$ & 153 \\
Hydraulic flow rate $\left(\mathrm{cm}^{3} / 30 \mathrm{~s}\right)$ & 320 \\
Hole number & 8 \\
Nozzle $k$-factor & 1.5 \\
\hline
\end{tabular}

without having parasitic side effects by nozzle seat throttling. The injector is a common-rail type actuated by a piezo-actuator. It allows the modelling of any kind of injection shape by varying the injection pressure via a piezo-actuated control valve at a fully opened nozzle needle. An additional advantage is a very fast opening and closing of the needle, which reduces the nozzle seat throttling effects to a minimum compared to current series production injectors. Detailed descriptions of the FIRST injector can be found in Schmüker' Specifications of the nozzle used are given in Table 1. See also Hinkelbein et al. ${ }^{8}$

\section{Realised combustion process}

The presented control system offers several new possibilities to adjust the injection. By not only adjusting the start of injection (SOI) or the duration of injection, but the whole combustion trace, a wide increase in degrees of freedom is gained. To fully utilise these new degrees of freedom, the setpoint curve also has to be defined in a way that not only single points, such as the start of combustion or the centre of combustion, are defined. Instead, a complete trace of setpoints has to be given to define the control target.

In addition to the well-known classical combustion processes for diesel engines, such as the Seiliger and the constant pressure process, a complete new class of processes can be generated with the new control system.
As a mixture of the Seiliger and constant pressure processes, the so-called $\alpha$-process is introduced.

This process comprises a constant pressure rise after the start of combustion at top dead centre (TDC). It can be expressed by the $\mathrm{CA} \Theta$ in the following equation

$$
w(i)=\alpha \cdot\left(\Theta_{i}-\Theta_{T D C}\right)+p_{C y l}\left(\Theta_{T D C}\right), \quad \forall i \geqslant i_{T D C}
$$

where the index $i$ gives the position of the CA after TDC. From equation (4), it can be seen that the complete pressure trace can be described with one characteristic parameter, which is the slope of the pressure rise $\alpha$. Although the trace is defined in a relative way based on the pressure trace of the motored engine, it can be recalculated for each control cycle to also take changes in engine load into account. Since the engine load is controlled by a separate governor, the constant increase of the pressure with increasing $\mathrm{CA}$, as described in equation (4), can be neglected. The end of the injection is controlled by the load governor and has therefore not to be considered in the setpoint equation. From equation (4), it can also be seen that the special case of $\alpha=0 \mathrm{bar} /{ }^{\circ} \mathrm{CA}$ is equal to the already known constant pressure process.

Figure 3 shows the definition of the $\alpha$-process as described in equation (4). The constant pressure rise starts at TDC and ends after the desired load is reached. At this point, the pressure trace changes into a normal expansion stroke.

The disadvantage of the relative determination of the setpoint vector is the limitation of describable sequences to a function depending on the CA. Processes with PPL can be described by an additional parameter $\Delta p$

$$
w_{P P L}(i)= \begin{cases}w(i) & \forall w(i)<p_{C y l}\left(\boldsymbol{\Theta}_{T D C}\right)+\Delta p \\ p_{C y l}\left(\boldsymbol{\Theta}_{T D C}\right)+\Delta p & \forall w(i) \geqslant p_{C y l}\left(\boldsymbol{\Theta}_{T D C}\right)+\Delta p\end{cases}
$$

and the setpoint profile from equation (4). By applying equation (5), the Seiliger process can be defined with an

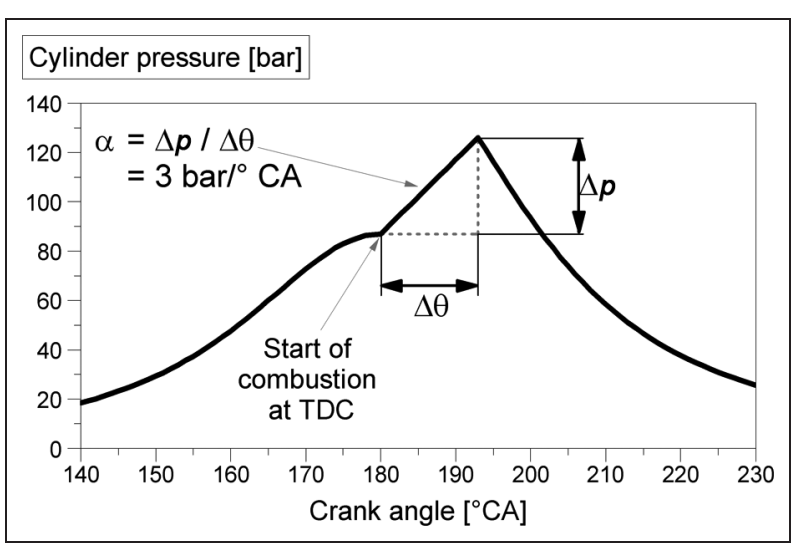

Figure 3. Definition of the $\alpha$-process. Operating point: 2280 $\mathrm{r} / \mathrm{min}, 10$ bar indicated mean effective pressure (IMEP). 
infinite $\alpha$ value and a PPL that fits to the corresponding Seiliger process. Therefore, the Seiliger process can be seen as a special case of the $\alpha$-process with PPL.

\section{Experimental results}

By feeding the two described setpoint traces of the $\alpha$-process and the $\alpha$-process with PPL to the control system, these processes can be obtained with the singlecylinder engine described in Table 1.

The results obtained after 50 control cycles are shown in Figure 4. The setpoint trace of the pure $\alpha$-process fits very well, whereas the $\alpha$-process with PPL shows some deviations at the beginning of the ramp. The figure also shows the required injection rates that are required to obtain the setpoint traces. These injection rates are automatically gained by the ILC algorithm. By comparing these two injection rates, it becomes obvious that the first part is nearly identical. Both start with a ramp-like injection rate, since the processes need to achieve a constant pressure rise of $4 \mathrm{bar} /{ }^{\circ} \mathrm{CA}$, starting at TDC. The injection rate of the pure $\alpha$-process starts to drop very fast after reaching a maximum of $2.1 \mathrm{mg} /{ }^{\circ} \mathrm{CA}$. Since a certain amount of time is required for the fuel to evaporate and ignite, the maximum of the injection rate is located a few ${ }^{\circ} \mathrm{CA}$ before the maximum of the cylinder pressure.

For the $\alpha$-process with PPL, the maximum of the injection rate is much lower and earlier than for the

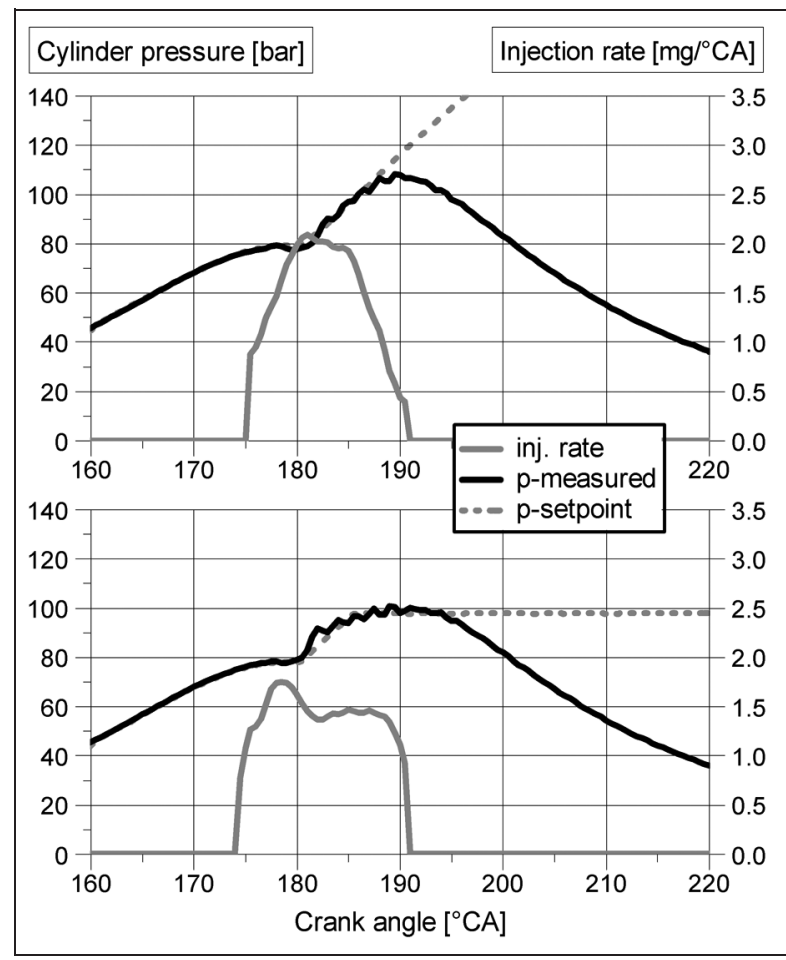

Figure 4. Experimentally realised $\alpha$-processes with slope of $4 \mathrm{bar} /{ }^{\circ} \mathrm{CA}$. Pure $\alpha$-process (top) and $\alpha$-process with PPL of 20 bar (bottom), both after 50 control cycles. Operating point: $2280 \mathrm{r} / \mathrm{min}, 9.4$ bar IMEP, 33\% EGR rate. pure $\alpha$-process. After the maximum is reached, the injection rate drops drastically and stays at this lower value to meet the PPL setpoint. For higher engine loads, the injection rate would start to increase again at the end of the injection to keep the cylinder pressure constant while the cylinder volume increases. However, for this load point, this effect is hardly noticeable. The difference at the beginning of the ramp results from an advanced injection timing of the $\alpha$-process with PPL to reach the overall best fit to the setpoint trace and not only to the ramp as for the pure $\alpha$-process.

How these two different injection rates (resulting from the different setpoint traces) influence the exhaust emissions of the engine can be seen in Figure 5. Here, an exhaust gas recirculation (EGR) variation with these two processes is shown. As seen in the top plot, the NOx-particulate trade-off of the pure $\alpha$-process is clearly lower than the one of the process with PPL. The pure $\alpha$-process almost reaches the EU 6 target values for this operating point. The target values are calculated from a 14 point test of a vehicle with $1590 \mathrm{~kg}$ mass and a 1.81 engine without a NOx-aftertreatment system for the new European driving cycle (NEDC).

The CO emissions of the pure $\alpha$-process also lie within the target range for EU 6. The process with PPL misses this target range and also shows a slight drawback in fuel consumption. As the fuel consumption is comparable for a wide range of EGR rates, the pure $\alpha$-process shows an advantage of approximately $1 \%$ for high EGR rates. The hydrocarbon emissions (not shown in Figure 5) are very low for both processes (less than $0.1 \mathrm{~g} / \mathrm{kWh}$ ) and therefore safely meet the EU 6 target.

However, the combustion sound level (CSL) (see Heuer ${ }^{10}$ ) shows a clear advantage for the process with PPL. The introduction of the PPL results in $1.5 \mathrm{dBA}$ less combustion noise. Both processes show a slight decrease in CSL with increasing EGR rate. To reduce the CSL, it is also possible to lower the slope of the pure $\alpha$-process, instead of introducing a PPL. Since the slope determines the pressure rise of the combustion, it is directly linked to the combustion sound. However, a lower slope also results in higher fuel consumption, and will lead to an increase in exhaust emission, especially in smoke emissions. By increasing the slope to values of, for example, $6 \mathrm{bar} /{ }^{\circ} \mathrm{CA}$, the fuel consumption approaches optimal values, but the CSL reaches values that are not acceptable for passenger car applications. This paper focuses on a slope of $4 \mathrm{bar} /{ }^{\circ} \mathrm{CA}$ since this slope gives the best trade-off regarding fuel consumption, exhaust emissions and CSL.

Since both investigated processes are variants of the $\alpha$-process, the question arises why the PPL results in such a clear drawback regarding the exhaust emissions of the engine. A first indication can be seen in Figure 4, which shows a big difference in required injection rates for both processes. How these different injection rates influence the exhaust emissions is analysed by performing combustion simulations with a three-dimensional 


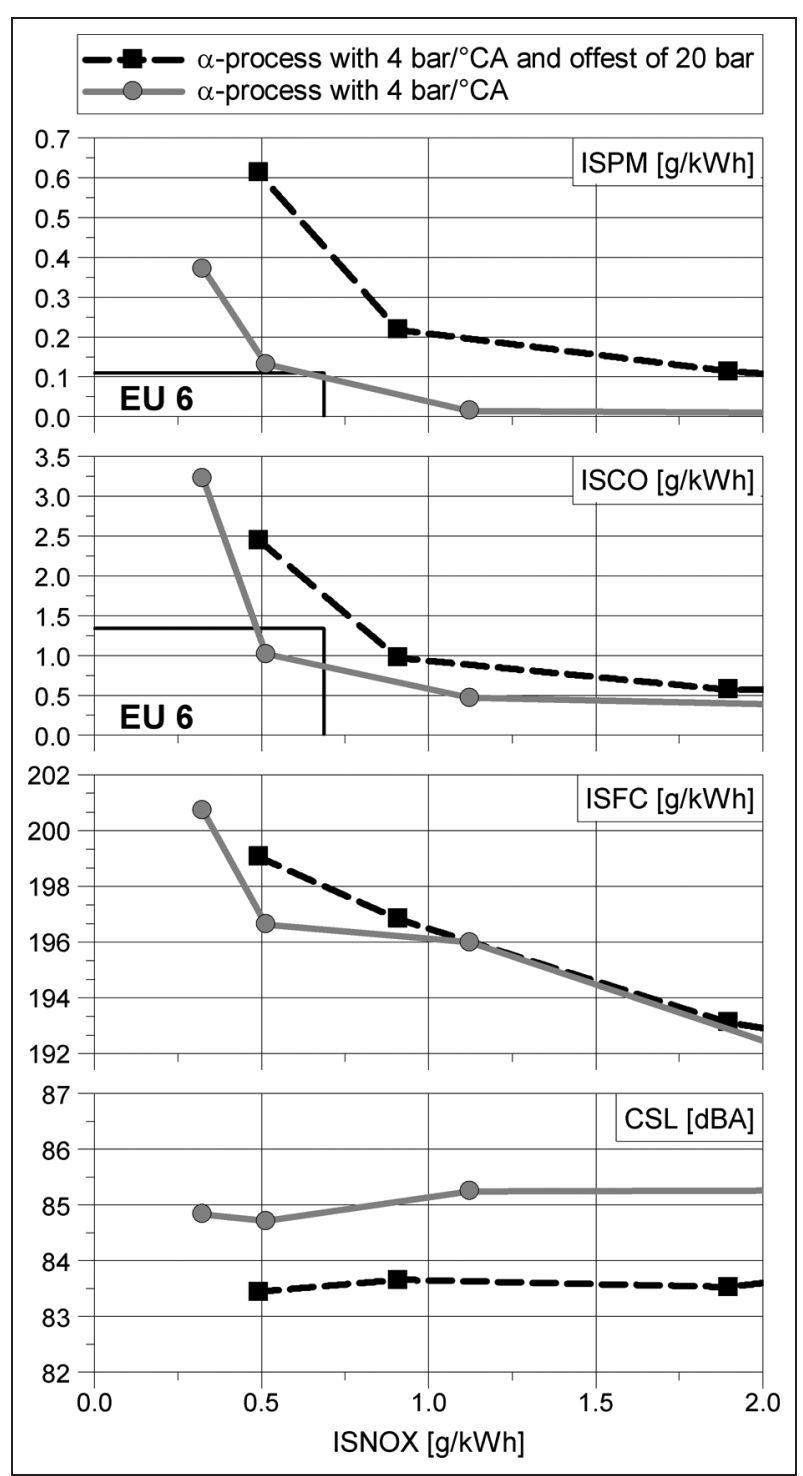

Figure 5. EGR variation of both $\alpha$-processes. Operating point: 2280 r/min, 9.4 bar IMEP (CSL: combustion sound level; ISCO: indicated specific $\mathrm{CO}$ emissions; ISFC: indicated specific fuel consumption; ISNOX: indicated specific NOx emissions; ISPM: indicated specific particulate matter).

(3D) computational fluid dynamics (CFD) tool. For the CFD analysis, the points of Figure 5 with the best NOx-particulate trade-off for each process were chosen. This is, in both cases, the measurement with an EGR rate of $33 \%$.

Of particular interest are the conditions in which soot is formed and oxidised to gain a better insight as to why the particulate emissions of the process with PPL are rather high. For this analysis, the 3D data of the CFD simulations are clustered into cells with soot forming conditions

$$
T \geqslant 1500 \mathrm{~K} \wedge \lambda \leqslant 0.6-\frac{T-1500 \mathrm{~K}}{3000 \mathrm{~K}}
$$

and cells with soot oxidation conditions

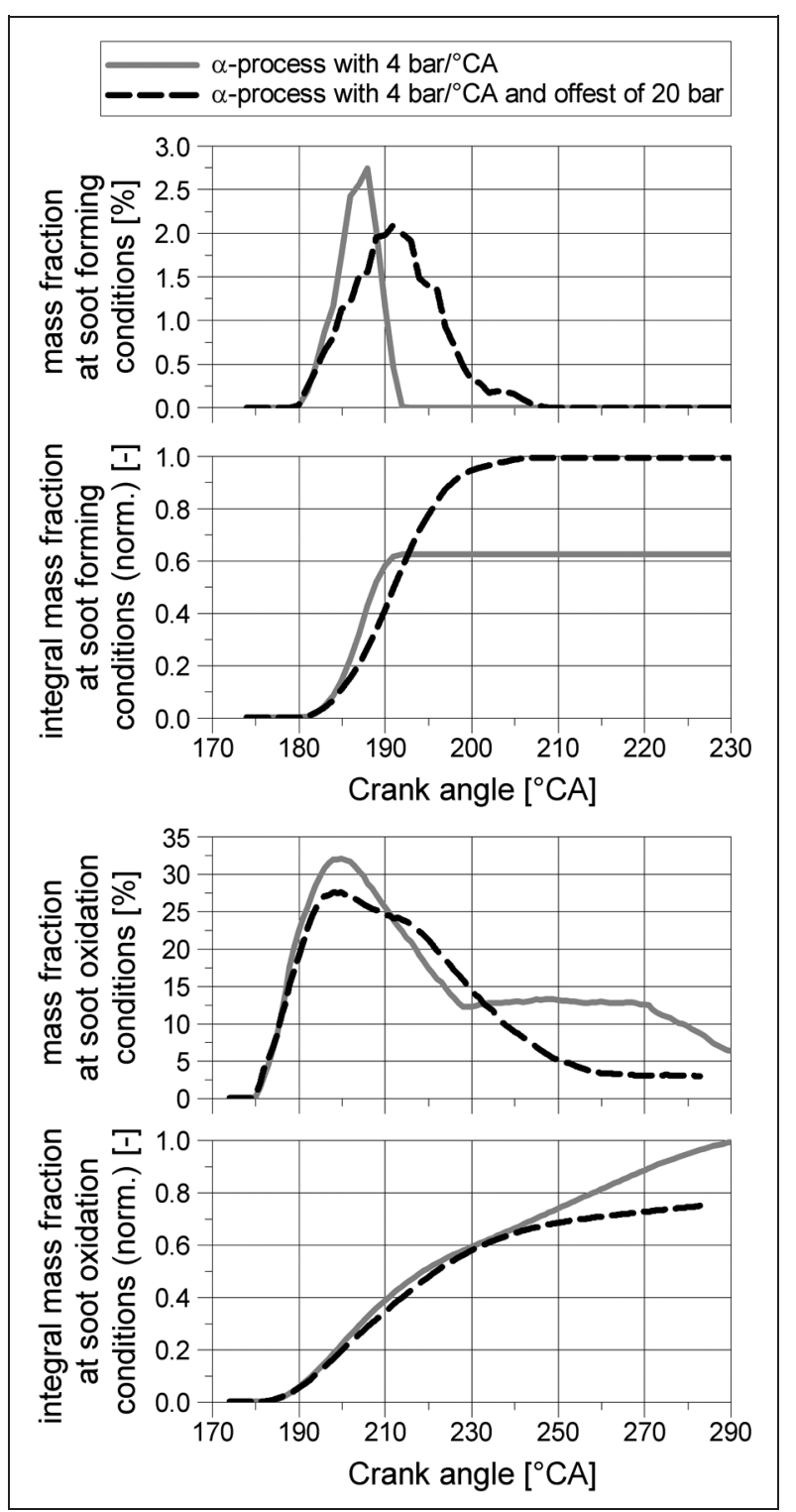

Figure 6. Mass fraction at soot forming and oxidation conditions. Operating point: $2280 \mathrm{r} / \mathrm{min}, 9.4$ bar IMEP, 33\% EGR rate.

$$
T \geqslant 1500 \mathrm{~K} \wedge \lambda \geqslant 1.1
$$

The clustering is carried out for each simulation step (in this case, each CA) separately by summing up the mass contained in these cells. The mass fraction, as well as the normalised integral of the mass fraction of these cells, versus the CA is shown in Figure 6. It can be seen that even with a higher peak value of mass at soot forming conditions, the integral of the pure $\alpha$-process is approximately $40 \%$ lower than that of the $\alpha$-process with PPL. This means that the process with PPL spends considerably more time at soot forming conditions. Additionally, the soot oxidation conditions are significantly better for the pure $\alpha$-process. In particular, at late CAs, the conditions are $20 \%$ more often reached than during the process with PPL. This clearly shows 


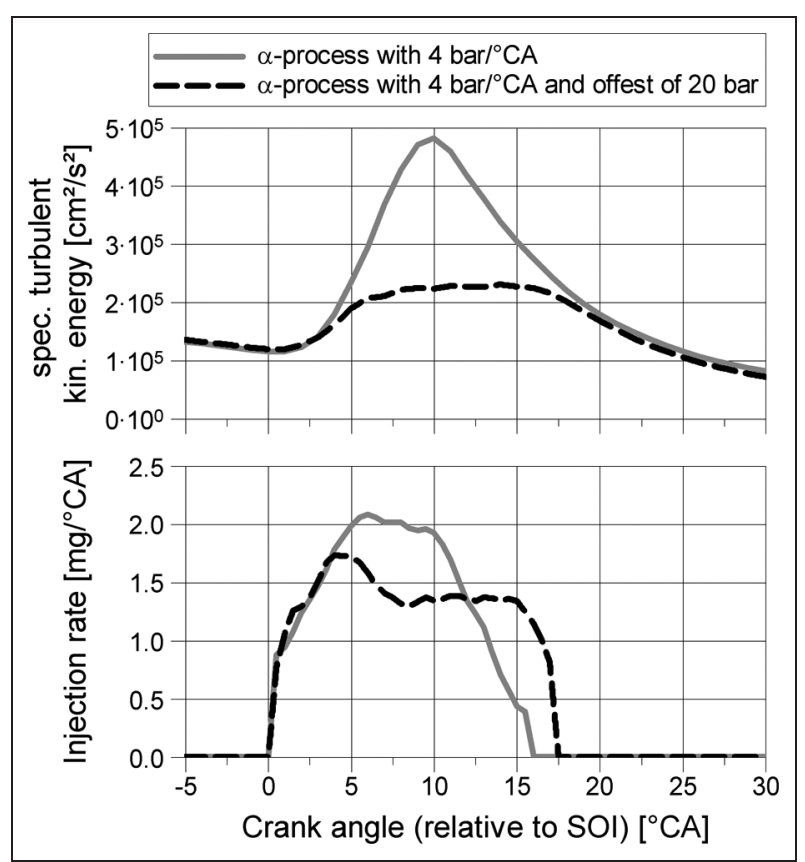

Figure 7. Specific turbulent kinetic energy and injection rate relative to SOI. Operating point: $2280 \mathrm{r} / \mathrm{min}, 9.4$ bar IMEP, 33\% EGR rate.

why the NOx-particulate trade-off of the $\alpha$-process with PPL is so disadvantageous.

The main reason for these drawbacks of the process with PPL is based on the different injection rates. By comparing the specific turbulent kinetic energy (Figure 7) of both processes, it becomes obvious that the peak energy of the pure $\alpha$-process is more than twice as high as that of the $\alpha$-process with PPL. This means that the mixture preparation is much better with the pure $\alpha$-process.

By comparing the turbulent kinetic energy with the injection rates (also shown in Figure 7), it can be seen that the difference is caused by the drop in injection rate that is needed to achieve the PPL. For the pure $\alpha$-process, the energy is constantly increasing until the end of the injection, whereas for the process with PPL, it stays almost constant after the peak of the injection rate is reached.

\section{Realisation of the control system with multiple injection strategies}

The following section presents an additional concept study that describes the potential of conventional highpressure diesel injectors to obtain predetermined pressure traces. Although the improvements regarding emissions and fuel consumption that can be achieved with the presented control system are high, a special flexible injector is necessary to control the combustion process. The high costs of the injection system automatically lead to the question of whether there is a way to also obtain predefined pressure traces with conventional injection systems. This can be carried out by applying strategies based on multiple injections. This approach is elaborated and discussed by using an adapted control strategy in combination with $3 \mathrm{D}$ CFD combustion simulations.

In order to use the ILC algorithm for the realisation of predefined pressure traces with multiple injection strategies, a number of adaptations have to be implemented. First of all, the discontinuous nature of the injection event has to be accounted for. Therefore, the injector voltage in its continuous form cannot be used as an actuating variable any more; the new actuating variable is a binary injector voltage in each sampling point, which turns on or off the injection (two-point control). This leads to a close dependency of the achievable results on the dynamical behaviour of the injector. The time scale on which an injection event can be executed has a direct influence on the performance of the control algorithm.

The modified control strategy is developed based on the above-described ILC structure. The general system setup and the data processing remain unchanged (see Figures 1 and 2); only the central equations describing the governor itself are adapted to the new boundary conditions, the basis of which is a two-point controller with hysteresis. As in the continuous case, each sampling point of the injection curve is related to a characteristic point of the pressure trace via an ignition delay model. ${ }^{8}$

The criteria for switching from active to inactive injection and vice versa are formulated with a proportional/differential scheme

$$
\begin{aligned}
& \underline{u}_{k+1}(i)= \\
& \left\{\begin{array}{ccc}
0 & \underline{e}_{k}(i)<-\delta_{\text {off, } P} \wedge \Delta \underline{e}_{k}(i)<-\delta_{\text {off }, D} \\
1 & \text { else } \\
1 & \underline{e}_{k}(i)>\delta_{\text {on, } P} & \wedge \Delta \underline{e}_{k}(i)>\delta_{\text {on, }, D} \\
0 & \text { else }
\end{array}\right\} \text { for } \underline{u}_{k}(i)=1 \\
& \text { for } \underline{u}_{k}(i)=0
\end{aligned}
$$

The actuating variable $\underline{u}_{k+1}$ is varied between active (1) and inactive (0), depending on the value of the control error $e_{k}$ (equation (2)) and the variation of the control error

$$
\Delta \underline{e}_{k}(i)=\frac{\underline{e}_{k}(i+1)-\underline{e}_{k}(i-1)}{2}
$$

Note that this differential definition is based on data points rather than time steps. The hysteresis threshold values for the proportional condition are indicated by $\delta_{o n, P}$ and $\delta_{o f f, P}$, and the thresholds for the differential condition by $\delta_{o n, D}$ and $\delta_{o f f, D}$. These thresholds are not constant over time, but are decreased stepwise when no change of the actuating variable $\underline{u}$ is detected between the two time steps $k$ and $k+1$. With this dependency of the control cycle, a gain scheduling scheme is introduced to the control algorithm. This is necessary in order to account for the unstable nature of two-point control algorithms and to overcome the trade-off between system stability at the beginning and small control deviations at the end of the control loop. As a 


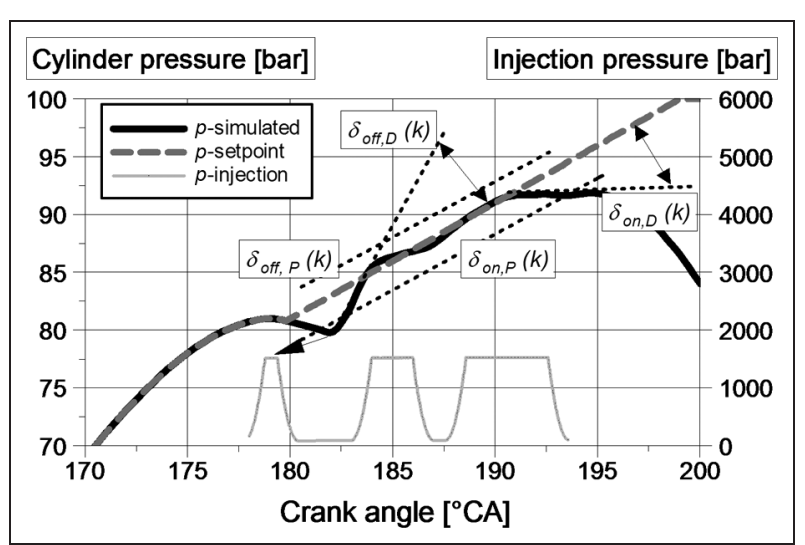

Figure 8. Controller setup with the corresponding proportional thresholds $\delta_{\text {on, } P}$ and $\delta_{\text {off, } \mathrm{B}}$ and differential thresholds $\delta_{\text {on, } D}$ and $\delta_{\text {off, D. }}$.

result, the actuating variable $\underline{u}_{k+1}$ is assigned a combination of time stamps and output values ( 0 or 1$)$ for each time stamp, which represents the injector actuation signal for the next cycle and is referred to as 'calculated injection profile'.

The implementation of these constraints is shown in Figure 8. Assuming a deactivated injection at a characteristic point of the pressure trace, the threshold value of the control error $\delta_{o n, P}$ and the threshold value of the variation of the control error $\delta_{o n, D}$ have to be exceeded in order to change the injection status to active at the related injection curve point. This case is indicated for the first injection in Figure 8. Since the proportional and differential criteria are exceeded at $182^{\circ} \mathrm{CA}$, injection number 1 will be activated in the next control cycle in order to meet the predetermined thresholds. This combination of proportional and differential error processing is suitable to control the predefined pressure trace. On the one hand, the proportional criterion directly prevents the cylinder pressure trace from deviating too far from the desired value. On the other hand, the differential criterion detects potential pressure rises and drops that might lead to a control error that cannot be compensated for by the proportional criterion. Also in Figure 8, the dependency of the threshold values $\delta_{o n, P}$ and $\delta_{o n, D}$ on the progress of the control event is expressed, as both are a function of $k$.

The so calculated actuating variable $\underline{u}_{k}(i)$ is furthermore verified by a logical check that processes the raw voltage curve with regard to the dynamical injector characteristics so that no technically impossible injector actuation is processed. This check contains the minimal distance between two injections, the minimal injection duration, as well as the imposition of reasonably shaped ramps at the beginning and end of the injections.

\section{Simulation results}

In order to analyse the adapted system, the control algorithm is coupled to a validated 3D CFD combustion model. The analysis is executed stepwise, and the

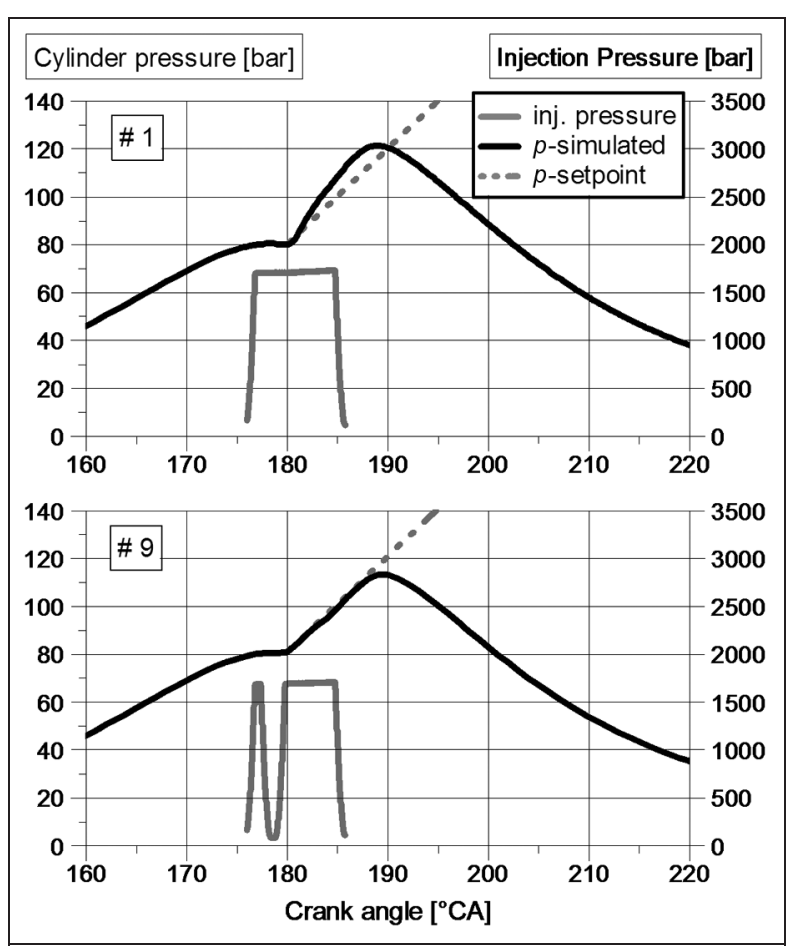

Figure 9. $\alpha$-process with $4 \mathrm{bar} /{ }^{\circ} \mathrm{CA}$ realised with the adapted control system. Control cycle \# I (top) and control cycle \# 9 (bottom). Operating point: $2280 \mathrm{r} / \mathrm{min}, 9.4 \mathrm{bar}$ IMEP.

calculated injection profile based on equation (8) is fed to the CFD model, which simulates the combustion with this particular injection. As a result, the corresponding pressure trace is transferred to the control algorithm, which compares this pressure trace with the setpoint vector and returns the next injection profile to the CFD model. This procedure is executed repeatedly until the stepwise reduction of the threshold values sets in and causes the control error to decrease with each control step. Each recalculation of the injection profile according to equation (8) is hereby considered a new control cycle.

Figure 9 shows the simulated combustion at the first control cycle of the control algorithm and after nine control cycles. Since the chosen $\alpha$ of $4 \mathrm{bar} /{ }^{\circ} \mathrm{CA}$ is close to the pressure gradient observed in that operating point without closed-loop combustion control, it only takes nine control cycles (e.g. nine variations of the injection profile until the predefined pressure trace is reached). In order to prevent the pressure trace from overshooting the setpoint curve in the area of $182^{\circ} \mathrm{CA}$, the injection is cut off at approximately $178^{\circ} \mathrm{CA}$ and reactivated shortly after. The length of the second injection is controlled to reach the desired load, as in the continuous case. From Figure 9, it can be seen that this automatically determined injection strategy meets the desired combustion trace accurately.

Although in Figure 9, only two injections are required to generate the desired combustion, the algorithm is capable of using as many as five injections or more, if necessary. This becomes essential as the 


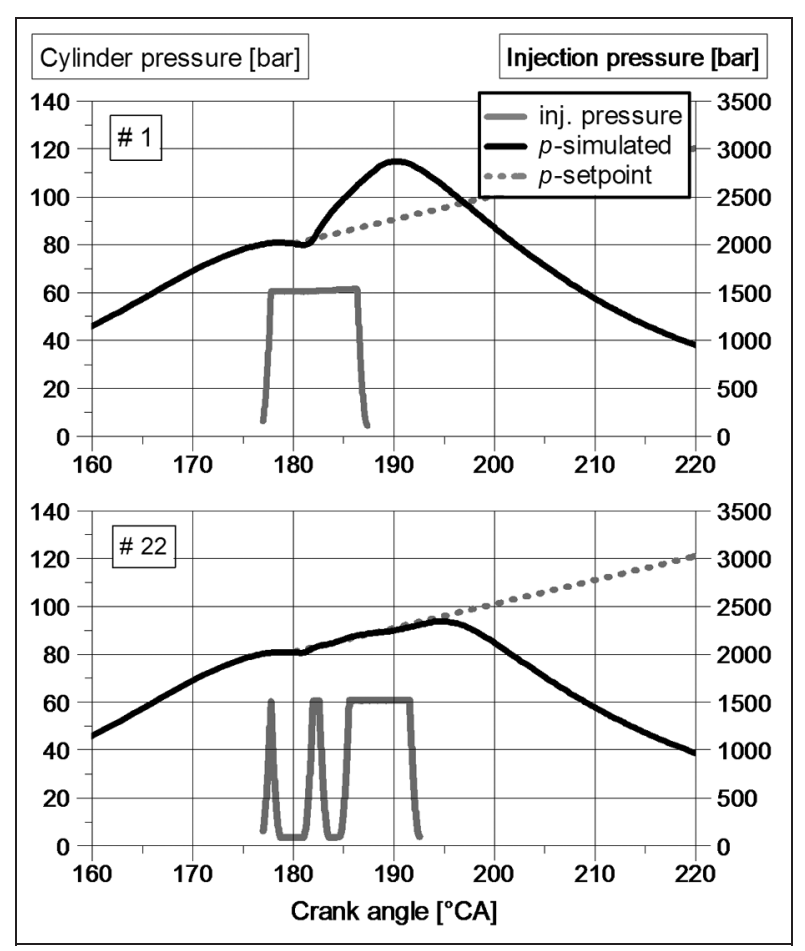

Figure 10. $\alpha$-process with I bar ${ }^{\circ} \mathrm{CA}$ realised with the adapted control system. Control cycle \# I (top) and control cycle \# 22 (bottom). Operating point: 2280 r/min, 10.9 bar IMEP.

predefined pressure trace differs more from the "natural' combustion in the considered operating point, as, for example, given by an $\alpha$-process with a ramp of $1 \mathrm{bar} /{ }^{\circ} \mathrm{CA}$. Since now the permitted pressure gradients are now much lower than in the previous case, an additional injection event is necessary in order to comply with the given thresholds, see Figure 10. To reach this injection profile, 22 control cycles are needed.

\section{Injector dynamics}

As already pointed out, in addition to a sophisticated control algorithm, the dynamical behaviour of the injector is also crucial for successful application of the multiple injection approach. This is why the injector characteristics need to be studied closely in order to identify the system-specific delay times and, in the next step, define the minimal acceptable delay times in order to gain a satisfyingly low overall final controller deviation. Therefore, an additional parameter study is executed. In this study, the above-mentioned logical check regarding the injector characteristics is varied to find out how a slowdown of the injector activation affects the control results obtained. The two parameters that are varied are the minimum time between two injections and the minimum total injection time. These two variables are considered to be characteristic for the dynamic behaviour of the injector. All simulations are executed at an engine speed of $2280 \mathrm{r} / \mathrm{min}$. Figure 11 shows the average control error from 0 to $15^{\circ} \mathrm{CA}$ after TDC as a function of the minimum time between two injections and the minimum total injection time. The

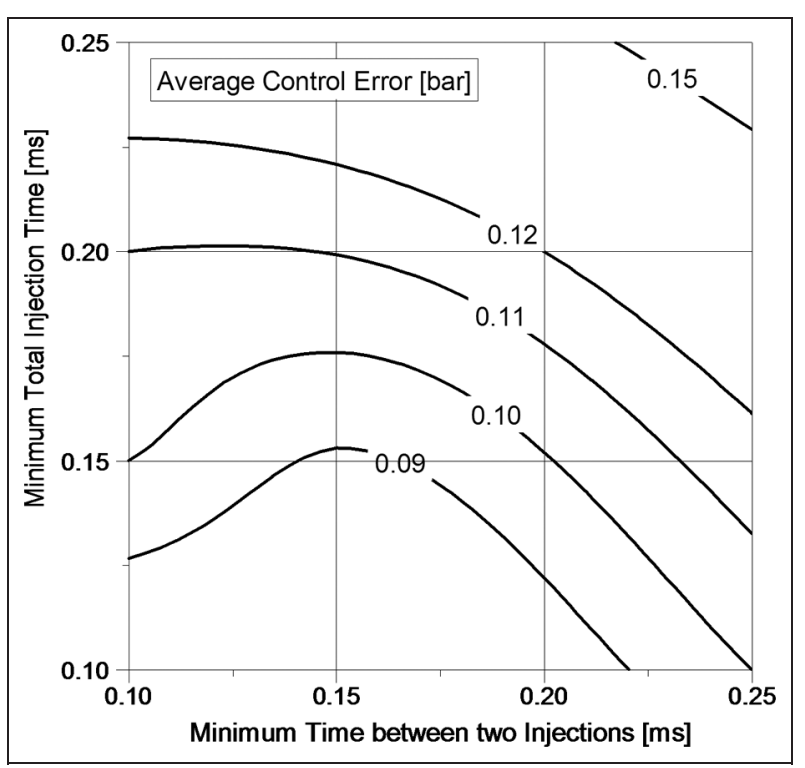

Figure II. Influence of the injector characteristics minimum total injection time (ms) and minimum time between two injections (ms) on the average control error (bar). Operating point: $2280 \mathrm{r} / \mathrm{min}, 9.4$ IMEP.

calculated value equals the averaged deviation between setpoint and actual cylinder pressure over the observed section of $15^{\circ} \mathrm{CA}$.

The analysis starts at a near-to-optimum value of $0.1 \mathrm{~ms}$ for both constraints (see Figure 11). This theoretical case is taken as the reference performance, expressed by an average control error of 0.08 bar. When the reaction speed of the injector is decreased, (e.g. both constraints are raised simultaneously), the average control error increases noticeably. In the most extreme case of $0.25 \mathrm{~ms}$ for both constraints, the error has doubled in comparison to the optimal starting point. However, these injector characteristics, which can be obtained with the newest generation of piezoactuated high-pressure diesel injectors, still supply an acceptable final control error.

When the two examined constraints are varied independently, another conclusion can be drawn. The minimum total injection time has a bigger effect on the final control error than the minimum time between two injection events. As indicated by the isolines along the $x$ - and $y$-axes of Figure 11, the effect of a minimum total injection time of $0.15 \mathrm{~ms}$ on the control error is equal to the effect of a minimum time between two injections of $0.25 \mathrm{~ms}$. The time needed to execute a complete injection event is therefore restrictive to the applicability of multiple injection strategies, rather than the minimum time between two injection events.

\section{Conclusion}

The presented paper outlines the development of a new system for closed-loop combustion control that uses the full potential of injection rate shaping. The control 
algorithm itself is based on ILC theory. To use the full potential of the control system, a new class of combustion processes, the so called $\alpha$-process, is introduced.

The detailed analysis of a pure $\alpha$-process and an $\alpha$-process with PPL shows clear disadvantages for the PPL, which is due to the decreasing injection rate at the end of the injection to reach the preset pressure limitation. From these results, it can be concluded that a PPL should be avoided as long as the mechanical boundary conditions of the engine or any optimisation targets for combustion noise require this limitation.

Additionally, a concept study is presented that proves the possibility of obtaining predefined pressure traces with multiple injections without using rateshaping capabilities. This concept provides the opportunity to exploit the described benefits of controlling the combustion pressure trace based on available series production injection systems. The technical constraints regarding the controller and the injection equipment are outlined and analysed. This analysis shows that injector constraints of $0.25 \mathrm{~ms}$ for both the minimum total injection time and the minimum time between two injections allow a stable realisation of closed-loop combustion control based on multiple injection strategies while satisfying final control errors. These technical characteristics can be obtained by modern, piezoactuated high-pressure diesel injectors. Experimental results of the adapted control system using this type of injectors and expansions for transient engine operation will be presented in the near future.

\section{Funding}

Parts of this work were performed as part of the Cluster of Excellence "Tailor-Made Fuels from Biomass", which is funded by the Excellence Initiative by the German federal and state governments to promote science and research at German universities. Additional information about the TMFB research approach is available at http://www.fuelcenter.rwth-aachen.de.

\section{References}

1. Albrecht W, Dohle U, Gombert R, et al. Innovative BOSCH common rail injection system CRSN4.2 for the new generation of DaimlerChrysler heavy duty dieselengines. Proceedings of the 28th Vienna Motor Symposium 2007: 162-180.

2. Desantes JM, Benajes J, Molina S, et al. The modification of the fuel injection rate in heavy-duty diesel engine. App Thermal Eng 2004; 24: 2701-2714.

3. Juneja H, Ra Y and Reitz RD. Optimization of injection rate shape using active control of fuel injection. SAE paper 2004-01-0530, 2004.

4. Rottmann M, Menne C, Pischinger S, et al. Injection rate shaping investigations on a small-bore DI diesel engine. SAE paper 2009-01-0850, 2009.

5. Arimoto S, Kawamura S and Miyazaki F. Bettering operation of robots by learning. $J$ Rob Sys 1984; 1(2): 123-140.
6. Rockel H. Analyse und Synthese parametrischer Iterativ Lernender Regelungen. Düsseldorf: VDI Verlag, 2006.

7. Pandit $\mathrm{M}$ and Buchheit K-H. Optimizing iterative learning control of cyclic production processes with application to extruders. IEEE Trans Contr Sys Tech 1999; 7(3): 382-390.

8. Hinkelbein J, Sandikcioglu C, Pischinger S, et al. Control of the diesel combustion process via advanced closed loop combustion control and a flexible injection rate shaping tool. SAE paper 2009-24-0114, 2009.

9. Schmücker J. Entwicklung eines piezoelektrischen Common-Rail Injektors mit variablem Einspritzverlauf. Dissertation, RWTH Aachen University, Germany, 2009.

10. Heuer S. Verbrennungsgeräusch des direkteinspritzenden Hubkolbenmotors. Dissertation, RWTH Aachen University, Germany, 2001.

\section{Appendix I}

\section{Notation}

$\underline{e}_{k} \quad$ vector of control error of control cycle $k$

$\underline{\mathrm{G}}$ matrix of plant, respectively, combustion

system, including injector

I identity matrix

$p \quad$ cylinder pressure (bar)

$\underline{S}$ matrix of the actuating variable filter

$\frac{\mathrm{S}}{T} \quad$ temperature $(\mathrm{K})$

$\underline{u}_{k} \quad$ vector of actuating variable of control cycle $k$

$\underline{w} \quad$ setpoint vector of the control system

$y_{k} \quad$ vector of feedback signal of control

cycle $k$

$\mathrm{z}^{-1} \quad$ unit delay

$\alpha$

cylinder pressure slope of the $\alpha$-process (bar ${ }^{\circ} \mathrm{CA}$ )

$\delta \quad$ allowed thresholds of the two-point control algorithm (bar)

$\lambda \quad$ lambda value

$\Theta \quad$ crank angle ( $\left.{ }^{\circ} \mathrm{CA}\right)$

I matrix of the learning operator

\section{Abbreviations}

CA crank angle

CFD computational fluid dynamics

CSL combustion sound level

EGR exhaust gas recirculation

FIRST flexible injection rate-shaping tool

ILC iterative learning control

IMEP indicated mean effective pressure

ISCO indicated specific $\mathrm{CO}$ emissions

ISFC indicated specific fuel consumption

ISNOX indicated specific NOx emissions

ISPM indicated specific particulate matter

NEDC new European driving cycle

PPL peak pressure limitation

RCP rapid control prototyping

SOI start of injection

TDC top dead centre 